\title{
Competitive match-play tennis under heat stress: a challenge for all players
}

\author{
Julien D Périard, ${ }^{1}$ Michael F Bergeron ${ }^{2}$
}

\section{HEAT STRESS IN TENNIS}

In January 2014, the heat took over centre court and the rest of the grounds during the first week of the Australian Open in Melbourne. Following days of scorching hot weather and air temperatures nearing $43^{\circ} \mathrm{C}$, play was suspended for several hours when the 'Extreme Heat Policy' was invoked. This entailed a stoppage in play, except that sets in progress had to carry on until completion, along with closing of the roofs over the arenas so that some matches could continue. The stoppage of play, however, occurred only after a plastic bottle had reportedly started melting on court, a ball boy and a male player fainted, a female player experienced cramping and vomiting and several notable players voiced their concerns regarding the appropriateness and safety of continuing to compete in such conditions. Further examination of the rules governing the suspension of play is seemingly warranted, in the context of balancing players' health and performance. This sequence of events played out on the international stage in Australia is mirrored each year around the world in numerous other much lower profile events, consistently and convincingly demonstrating the challenges and consequences of competing under excessive heat stress. Arguably, the current guidelines and practices for effectively and safely playing tennis in the heat, or the thresholds to suspend play, may not be adequate and sufficiently evidence based.

Competitive match-play tennis is multifactorial, underpinned by an integrated array of complex physiological, biomechanical, cognitive and psychological processes. Players must have an appropriate balance of particular physical attributes such as speed, agility, power, muscular endurance and a high level of overall aerobic fitness, as well as a keen-sustained capacity to constantly anticipate, quickly

\footnotetext{
${ }^{1}$ Athlete Health and Performance Research Centre Aspetar, Qatar Orthopaedic and Sports Medicine Hospital, Doha, Qatar; ${ }^{2}$ National Youth Sports Health \& Safety Institute, Department of Pediatrics, Sanford USD School of Medicine, Sioux Falls, South Dakota, USA

Correspondence to Dr Julien Périard, Aspetar, Qatar Orthopaedic and Sports Medicine Hospital, Research and Education Centre, PO Box 29222, Doha, Qatar; julien.periard@aspetar.com
}

react and make numerous ongoing splitsecond decisions. ${ }^{1}$ When undertaken in hot and/or humid conditions, all of these essential processes are challenged, with further increasing levels of cardiovascular and thermal strain ultimately affecting performance and potentially unduly impacting a player's well-being.

Tennis has been contested outdoors for some time now; so, having to contend with challenging environmental conditions, including the heat, is nothing new. What has changed is, however, notable. The more fit and muscular players of today compete with incredible racquet and string technology and a seemingly everincreasing level of intensity and power. The pace of play has also been accelerated by mandated shorter intervals between points. With the growth of the game worldwide, tennis can now be played yearround in warm-to-hot climates in previously unthinkable locations, where deserts (eg, Indian Wells and Doha) are turned into world class facilities and luxury tennis resorts, with stadiums and surrounding courts basking in the sun's relentless electromagnetic energy. All of these factors contribute to greater heat exposure and thermal strain for tennis players. Countermeasures have been developed and are widely utilised-for example, more breathable clothing, improved hydration practices and a variety of on-court cooling measures and strategies. Nevertheless, heat-related problems continue at all levels of competitive play.

\section{IN THIS ISSUE}

In this themed supplement issue, Aspetar, Qatar Orthopaedic and Sports Medicine Hospital, partners with BJSM to address the topic of match-play tennis from the perspective of the various influences of environmental heat stress. The issue examines how hyperthermia and hydration have the potential to impact a player's health, performance and safety. Through targeted reviews and original investigations, environmental heat, hydration and physiological strain are explored; consequent effects on performance, fatigue and recovery are examined and popular fitness testing protocols are addressed.
Targeted reviews

The first review by Reid and Duffield ${ }^{2}$ examines the various pathways by which fatigue develops during match-play tennis. Specifically, the authors discuss how physiological, mechanical and psychological responses affect performance during prolonged matches. They highlight the need for research that reflects the specific demands of competitive match play in the upper echelons of the sport, and the necessity for studies that examine alterations in physiological and neuromuscular function in the context of their influence on technical, decision-making and movement skills.

During competitive match-play tennis in the heat, a measurable rise in thermal strain and the consequent exacerbated loss of body water through extensive sweating can challenge a player's physiology, perception of effort, performance and health. Given these recognised challenges, especially in tournament settings, Bergeron ${ }^{3}$ provides a thorough review of the role of hydration and thermal strain during competitive match-play tennis in the heat. The author outlines key recent findings featuring hydration-related measures and thermal strain during high-level tennis training and competition under heat stress. Issues relating to scheduling and repeated same-day matches, concerns regarding selected clinical conditions and medications, as well as caffeine, are also discussed, and the question 'Where do we go from here?' is candidly addressed. Finally, key recommendations of best practices to minimise exertional heat illness risk and improve player safety, well-being and on-court performance are prominently emphasised.

Irrespective of the environmental conditions, it is well established that prolonged match-play tennis and training result in the development of measureable fatigue. Accordingly, Kovacs and Baker ${ }^{4}$ examine recovery interventions and strategies to improve tennis performance by limiting the severity of fatigue and/or accelerating recovery. The review focuses on four recovery techniques commonly used in tennis to improve adaptation and performance; namely, temperature-based interventions, compressive clothing, electronic interventions and nutritional interventions.

The final targeted review by FernandezFernandez et $a l^{5}$ addresses the topic of fitness testing for tennis players. The game has evolved from a sport in which skill was the primary pre-requisite for success to a sport that requires much greater physical, physiological and psychological attributes to effectively compete and 
sustain high-level performance. Accordingly, fitness testing at all levels of play has become a more prevalent and expected practice in determining a player's capacity to perform and a benchmark for gauging effectiveness of training and individual improvement. The authors describe and evaluate the various physical tests recommended and used by practitioners and sports scientists in the laboratory and field settings. Furthermore, the most appropriate tests are explained and respective age and gender normative values are provided.

\section{Original investigations and novel findings}

The original investigations highlighted in the supplement stem from an extensive study conducted at Aspetar over the period of a month with high-level European tennis players ranked from 1 to 3 by the International Tennis Federation (ITF). This comprehensive research project evaluated the influence of heat stress and hydration on match-play tennis performance in a field setting with a multidisciplinary approach.

The first article contrasts the thermal, physiological and perceptual responses associated with match-play tennis in hot and cool conditions, along with the accompanying adjustments in match characteristics. ${ }^{6}$ The results suggest that adjustments in match-play characteristics (ie, an increase in time between points leading to a decrease in effective playing time) under severe heat stress represent a behavioural strategy adopted to minimise or offset the sensation and effects of environmental conditions being rated as difficult. In the second manuscript, the time course of changes in physical performance in response to match-play tennis in the heat is assessed. ${ }^{7}$ It is noted that the physical performance parameters (ie, sprinting and jumping) associated with matchrelated fatigue are primarily impaired after $\sim 60 \mathrm{~min}$ of total playing time (ie, $10 \mathrm{~min}$ of effective play), with limited additional reductions thereafter, and that full recovery occurs within $24 \mathrm{~h}$. A novel finding is that the additional increase in body core temperature incurred in the hot compared with the cool match did not exacerbate the observed reduction in physical performance.

From a neuromuscular perspective, impairments in lower limb strength and voluntary activation are highlighted in the third article in this series, ${ }^{8}$ whereas the fourth study assessed the time course of changes in rapid muscle torque production capacity. ${ }^{9}$ The results indicate that knee extensor and plantar flexor strength is reduced following match-play tennis in hot and cool conditions. Interestingly, the reduction in strength was exacerbated under heat stress in the knee extensors, and remained depressed for $24 \mathrm{~h}$ after match completion. The loss of strength was ascribed to a combination of central and peripheral fatigue factors in the knee extensors, and mainly to peripheral adjustments in the plantar flexors. In the knee extensors, the reduction in strength appeared to also account for a decrease in the rate of torque development, irrespective of the environmental conditions.

Article five compares the impact of match-play tennis in hot and cool conditions on changes in oxidative stress and antioxidant status. $^{10}$ Intriguingly, it appears that match play in the heat does not exacerbate the development of oxidative stress, but rather significantly increases antioxidant status. This suggests that the heat stress may provide a necessary signal for the upregulation of antioxidant defence, thus dampening cellular damage.

The final investigation of the supplement examined whether an individualised hydration regimen reduces thermal, physiological and perceptual strain during match-play tennis in the heat, and minimises the alterations in physical performance and neuromuscular function postmatch and into recovery. ${ }^{11}$ The results indicate that an individualised hydration regimen and ad libitum fluid consumption allow for minimal body mass losses $(<1 \%)$ to occur during match-play tennis in the heat. However, undertaking play in a well-hydrated state does appear to attenuate the early rise in thermal, physiological and perceptual strain. Conversely, repeated-sprint ability and maximal voluntary strength in the lower limbs deteriorated similarly following play under heat stress, regardless of whether competition was undertaken in a euhydrated or slightly dehydrated state, but recover within $24 \mathrm{~h}$.

From the findings highlighted in this supplement issue, it is clear that competitive match-play tennis in the heat can lead to significantly greater levels of thermal strain (ie, body core temperature $>39.5^{\circ} \mathrm{C}$ and elevated skin temperature) compared with moderate or cool conditions. This occurs despite the ability to hydrate adequately (ie, body mass losses generally $\leq 1 \%)$ and mitigate excessive thermal strain by employing self-pacing and on-court cooling strategies. Any measurable increase in overall thermal strain leads to a cascade of alterations in match characteristics, neuromuscular function, oxidative stress and antioxidant status, which can impact on performance acutely and potentially more so in successive matches during tournament play.

This 2014 supplement issue of the BJSM measurably extends the limited data and the current perspectives regarding competitive match-play tennis in the heat. The novel research and reviews featured in this supplement are important new contributions that significantly advance our knowledge and appreciation of the physiological responses in tennis players and the related on-court and recovery challenges they face with training and competing in hot and/or humid conditions. While these new studies have contributed to enhance our knowledge and appreciation regarding competitive matchplay tennis in the heat, they, along with the targeted reviews, have also provided a number of relevant questions that remain to be explored. However, the future is bright. Particularly with the advent of new monitoring and tracking technologies, in the years ahead, tennis research in adverse environmental field conditions will be extremely interesting and likely provide many new insights and opportunities to further enhance on-court wellbeing, safety and performance for all players.

Contributors JDP and MFB had intellectual input in drafting the article and gave final approval for submitting the manuscript.

Funding This project was funded by the Aspire Zone Foundation Research Grant.

Competing interests None.

Provenance and peer review Not commissioned; internally peer reviewed.
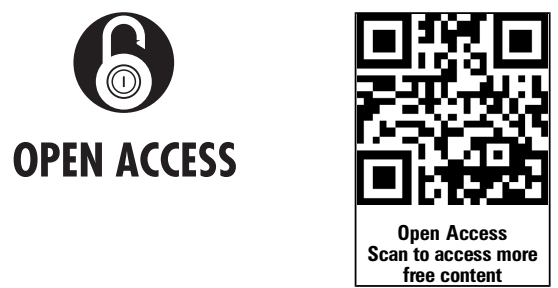

Open Access This is an Open Access article distributed in accordance with the Creative Commons Attribution Non Commercial (CC BY-NC 3.0) license, which permits others to distribute, remix, adapt, build upon this work non-commercially, and license their derivative works on different terms, provided the original work is properly cited and the use is noncommercial. See: http://creativecommons.org/licenses/ by-nc/3.0/

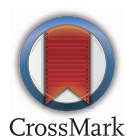

To cite Périard JD, Bergeron MF. Br I Sports Med 2014:48:i1-i3. 
Accepted 29 January 2014

Br J Sports Med 2014;48:i1-i3.

doi:10.1136/bjsports-2014-093496

\section{REFERENCES}

1 Hornery DJ, Farrow D, Mujika I, et al. Fatigue in tennis: mechanisms of fatigue and effect on performance. Sports Med 2007;37:199-212.

2 Reid M, Duffield R. The development of fatigue during match-play tennis. Br J Sports Med 2014;48: i7-11.

3 Bergeron MF. Hydration and thermal strain during tennis in the heat. Br J Sports Med 2014;48: i12-17.
4 Kovacs MS, Baker LB. Recovery interventions and strategies for improved tennis performance. $\mathrm{Br} J$ Sports Med 2014;48:i18-21.

5 Fernandez-Fernandez J, Ulbricht A, Ferrauti A. Fitness testing of tennis players. Br I Sports Med 2014;48: i22-31.

6 Périard JD, Racinais S, Knez W, et al. Thermal, physiological and perceptual strain mediate alterations in match-play tennis under heat stress. Br J Sports Med 2014:48:i32-8.

7 Girard O, Christian RJ, Racinais S, et al. Heat stress does not exacerbate tennis-induced alterations in physical performance. Br J Sports Med 2014;48: i39-44.

8 Périard JD, Girard O, Racinais S. Neuromuscular adjustments of the knee extensors and plantar flexors following match-play tennis in the heat. $\mathrm{Br} J$ Sports Med 2014;48:i45-51.

9 Girard O, Racinais S, Périard JD. Tennis in hot and cool conditions decreases rapid muscle torque production capacity in the knee extensors but not the plantar flexors. Br I Sports Med 2014;48: i52-8.

10 Knez W, Périard JD. The impact of match-play tennis in a hot environment on indirect markers of oxidative stress and antioxidant status. Br I Sports Med 2014;48:i59-63.

11 Périard JD, Racinais S, Knez W, et al. Coping with heat stress during match-play tennis: does an individualized hydration regimen enhance performance and recovery? Br J Sports Med 2014;48:164-70. 